\title{
Phase-coherence and symmetry in four-terminal magnetotransport measurements on InN nanowires
}

R. Frielinghaus, S. Estévez Hernández, R. Calarco, and Th. Schäpers

Citation: Appl. Phys. Lett. 94, 252107 (2009);

View online: https://doi.org/10.1063/1.3159626

View Table of Contents: http://aip.scitation.org/toc/apl/94/25

Published by the American Institute of Physics

\section{Articles you may be interested in}

Universal conductance fluctuations and localization effects in InN nanowires connected in parallel Journal of Applied Physics 108, 113704 (2010); 10.1063/1.3516216

Temperature dependence of the phase-coherence length in $\ln N$ nanowires Applied Physics Letters 92, 132101 (2008); 10.1063/1.2905268

Spin accumulation at in-situ grown Fe/GaAs(100) Schottky barriers measured using the three- and fourterminal methods

Applied Physics Letters 109, 122409 (2016); 10.1063/1.4963281

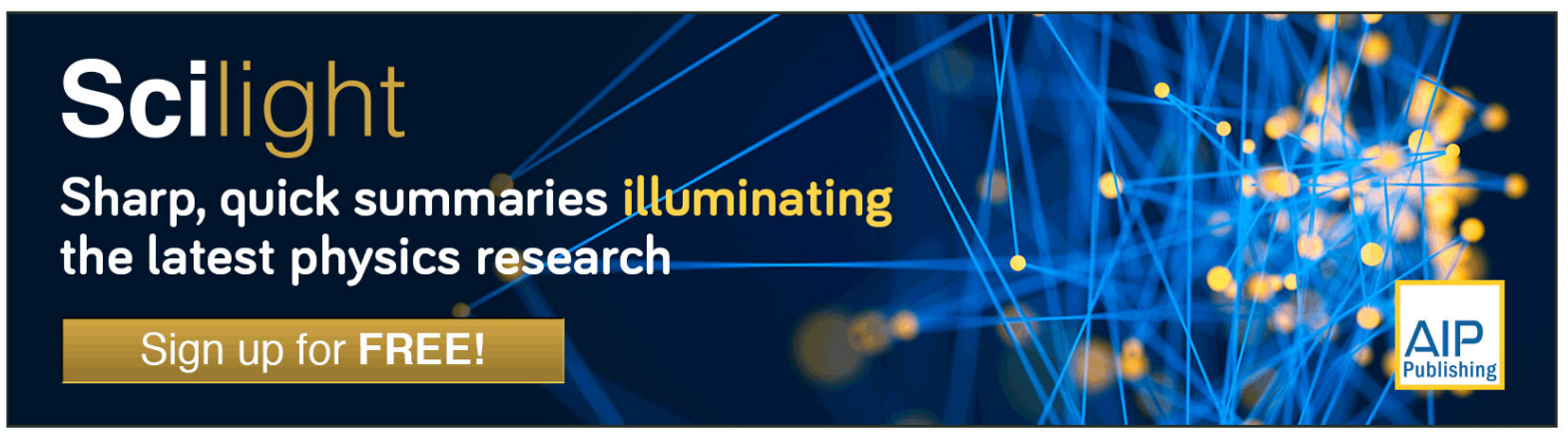




\title{
Phase-coherence and symmetry in four-terminal magnetotransport measurements on InN nanowires
}

\author{
R. Frielinghaus, S. Estévez Hernández, R. Calarco, and Th. Schäpers ${ }^{\text {a) }}$ \\ Institute of Bio- and Nanosystems (IBN-1) and JARA-Fundamentals of Future Information Technology, \\ Research Centre Jülich GmbH, 52425 Jülich, Germany
}

(Received 5 April 2009; accepted 4 June 2009; published online 25 June 2009)

\begin{abstract}
Universal conductance fluctuations in $n$-type doped InN nanowires are investigated at temperatures down to $0.35 \mathrm{~K}$. The nanowires were grown by molecular beam epitaxy. The effect of the contact resistance is eliminated by performing the measurements in a four-terminal configuration. We find that the decrease in the conductance fluctuation amplitude with temperature is due to small energy transfer phase-breaking processes and thermal broadening. In contrast to measurements in a two-terminal configuration, the symmetry of the conductance under magnetic field reversal is lost. (C) 2009 American Institute of Physics. [DOI: 10.1063/1.3159626]
\end{abstract}

Semiconductor nanowires are very interesting candidates for the realization of future nanoelectronic devices. ${ }^{1,2} \mathrm{Be}-$ sides this application-driven research, nanowires are also ideal systems to study fundamental quantum effects, i.e., single electron tunneling or phase-coherent transport. ${ }^{3-7}$ Among the III-V semiconductors, $\mathrm{InN}$ is a particularly interesting material for nanowires because of its low band gap and its surface accumulation layer. ${ }^{8-11}$ This ensures a large electron concentration, a high conductivity, and Schottky barrier-free contacts. The low temperature transport in nanowires is affected to a large extent by electron interference effects. In small size conductors such as semiconductor nanowires, phase-coherent transport manifests itself by the appearance of universal conductance fluctuations. ${ }^{12,13}$ Upon analyzing these fluctuations as a function of temperature, valuable information on the phase-coherence of the electrons can be obtained.

We measured universal conductance fluctuations in $n$-type doped InN nanowires in order to study the phasecoherence in the electron transport. In contrast to previous investigations, the measurements are performed in a fourterminal configuration, which allows to eliminate contributions of the contact resistances. By a direct comparison of two- and four-terminal measurements, we are able to assess the reliability of two-terminal measurements for the study of phase-coherent transport. Moreover, the difference in the symmetry of the magnetoconductance under field reversal in both measurement configurations will be discussed in detail.

The $n$-type doped $\mathrm{InN}$ nanowires were grown on a $\mathrm{Si}$ (111) substrate by plasma-assisted molecular beam epitaxy (MBE). For the $n$-type doping Si was used. The resulting wires have diameters between 90 and $130 \mathrm{~nm}$ with a length up to $2.5 \mu \mathrm{m} .{ }^{10}$ For electrical characterization the wires were removed mechanically from the original substrate in an acetone solution. Subsequently, they were placed on a $\mathrm{Si}$ (100) wafer covered with a $100 \mathrm{~nm}$ thick $\mathrm{SiO}_{2}$ insulating layer. By using electron beam lithography each wire was contacted individually with four nonalloyed Ti/Au Ohmic electrodes. ${ }^{6}$ Most of the measurements reported here were performed on one representative nanowire (sample A). The corresponding scanning electron micrograph is shown in the

${ }^{a)}$ Electronic mail: th.schaepers@fz-juelich.de. inset of Fig. 2(a). The wire has a diameter of $d=95 \mathrm{~nm}$. From contacts 1-4 the length of the wire segments between the contact pads are 280, 455, and $295 \mathrm{~nm}$, respectively. At a temperature of $0.5 \mathrm{~K}$ the following contact resistances were obtained for contacts 1-4: 1090, 930, 230, and $440 \Omega$, respectively. From resistance measurements in a four-terminal configuration a resistivity of $\rho_{3 D}=180 \mu \Omega \mathrm{cm}$ was determined, which is by a factor of about 3 lower than for undoped $\mathrm{InN}$ nanowires. If one assumes that the mobility is not changed significantly with carrier concentration $n$, one can estimate $n$ to be about $1.5 \times 10^{19} \mathrm{~cm}^{-3}$ being a factor of 3 higher than the values reported for nominally undoped $\mathrm{InN}$ nanowires. ${ }^{10}$ Using this value for $n$, the mobility $\mu$ and diffusion constant $\mathcal{D}$ are calculated to be $2300 \mathrm{~cm}^{2} / \mathrm{V} \mathrm{s}$ and $4.8 \times 10^{-6} \mathrm{~cm}^{2} / \mathrm{s}$, respectively. For comparison we also present some measurements of a second nanowire (sample B). This wire had a diameter of $100 \mathrm{~nm}$ and a length of the center segment of $320 \mathrm{~nm}$.

Transport measurements were performed in a He-4 flow cryostat from room temperature down to $4 \mathrm{~K}$ and magnetotransport measurements in a He-3 cryostat at temperatures between 0.35 and $25 \mathrm{~K}$. The resistance was measured by using a standard lock-in technique with an ac bias current of $60 \mathrm{nA}$. The magnetic field was oriented perpendicularly to the wire axis.

In Fig. 1(a) the temperature dependence of the fourterminal resistance of sample A is plotted. The corresponding measurement configuration is depicted in Fig. 3(d) (inset). Down to about $150 \mathrm{~K}$, the decrease in the resistance can be expressed by a linear temperature dependence $R(T)=R_{0}\left(T_{0}\right)$ $\times\left[1+\alpha\left(T-T_{0}\right)\right]$, with $R_{0}\left(T_{0}\right)=278 \Omega$, the resistance at $T_{0}$ $=273 \mathrm{~K}$, and $\alpha=5 \times 10^{-4} \mathrm{~K}^{-1}$. At temperatures below 150 $\mathrm{K}$ the resistance tends to saturate at a value of about $230 \Omega$. The observed temperature dependence is characteristic for a metal-like degenerate electron gas in the $\mathrm{InN}$ nanowire. The results obtained here are in accordance with previous measurements on $\mathrm{InN}$ nanowires performed in a two-terminal configuration. ${ }^{7}$ The comparison between both measurement configurations confirms our former assumption that the temperature dependence can be attributed to the $\mathrm{InN}$ nanowire itself while the effect of the temperature on the contact resistance is negligible. 

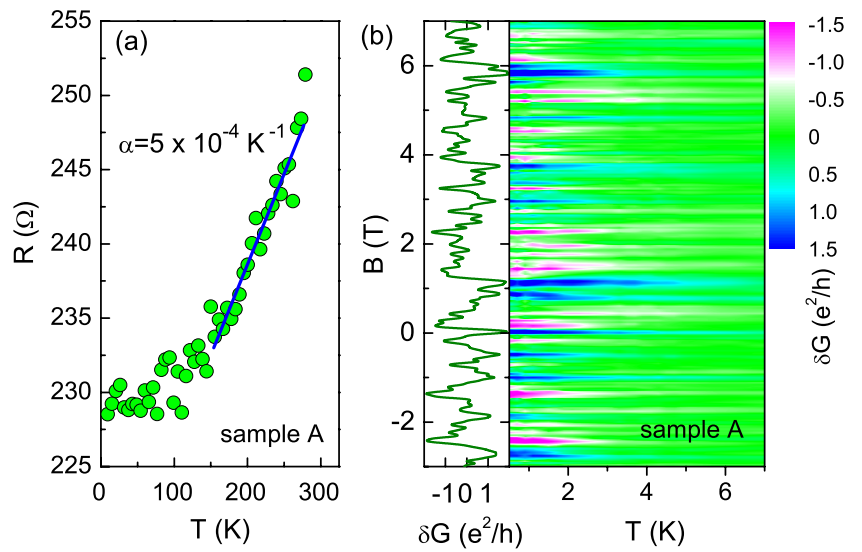

FIG. 1. (Color online) (a) Resistance of sample A as a function of temperature measured in a four-terminal configuration. The solid line represents a linear fit at higher temperatures. (b) Normalized conductance fluctuations of sample $\mathrm{A}$ in units of $e^{2} / h$ as a function of magnetic field and temperature. The curve on the left side shows the conductance fluctuations at $0.35 \mathrm{~K}$.

At low temperatures the four-terminal magnetoconductance fluctuates as a function of magnetic field. The fluctuations $\delta G=G-G_{0}$ were determined by subtracting the average conductance $G_{0}$. As can be seen in Fig. 1(b), the fluctuation pattern is reproducible while its amplitude decreases with increasing temperature. The varying magnetoconductance $\delta G$ can be attributed to universal conductance fluctuations. $^{12,13}$ As can be seen in Fig. 2(a), up to about $1.2 \mathrm{~K}$ the root-mean-square $\operatorname{rms}(\delta G)$ of the fluctuation amplitude decreases slowly with temperature, while at temperatures higher than $1.2 \mathrm{~K}$ a much steeper decrease proportional to $T^{-0.84}$ is observed. As will be explained in detail below, the slow decrease in $\operatorname{rms}(\delta G)$ at temperatures below $1.2 \mathrm{~K}$ can be attributed to the fact that here the phase-coherence length $l_{\phi}$ is comparable to the separation of the inner pair of contacts. The steeper decrease at higher temperatures can be attributed to two contributions. First, to the thermal broaden-
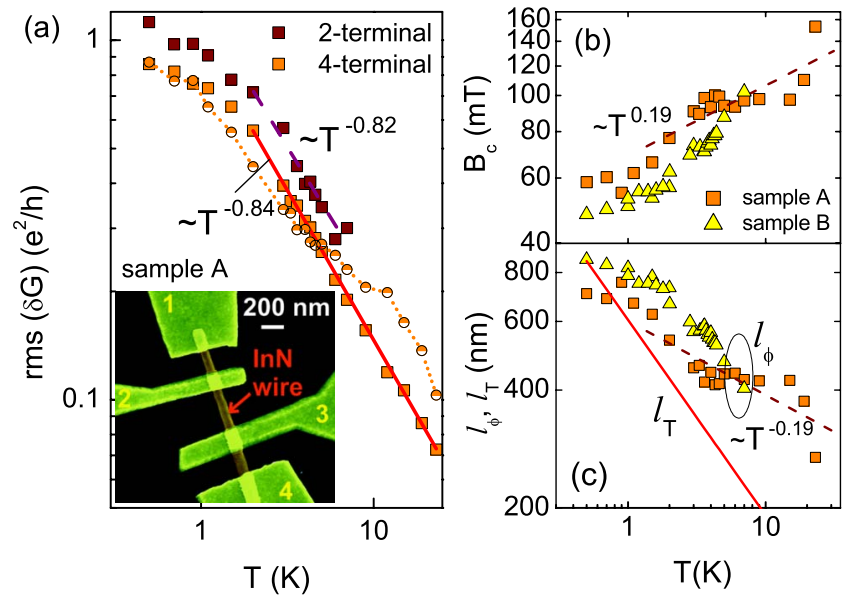

FIG. 2. (Color online) (a) $\mathrm{rms}(\delta G)$ vs $T$ of sample A measured in a two- and four-terminal configurations (squares), respectively. The solid lines represent the fitted temperature dependence. Also shown are the rms values obtained from Eq. (1) by using $l_{\phi}$ extracted from $B_{c}$ (dots). The inset shows an electron beam micrograph of the sample. (b) $B_{c}$ vs $T$ of sample A (squares). Above $1.5 \mathrm{~K} B_{c}$ increases as $T^{0.19}$ (dashed line). For comparison $B_{c}$ determined for sample B is also shown (triangles). (c) $l_{\phi}$ determined from $B_{c}$ for sample A (squares) and sample B (triangles), respectively. The dashed line represents the fitted decrease in $l_{\phi}$ for sample A while the solid line represents $l_{T}$.
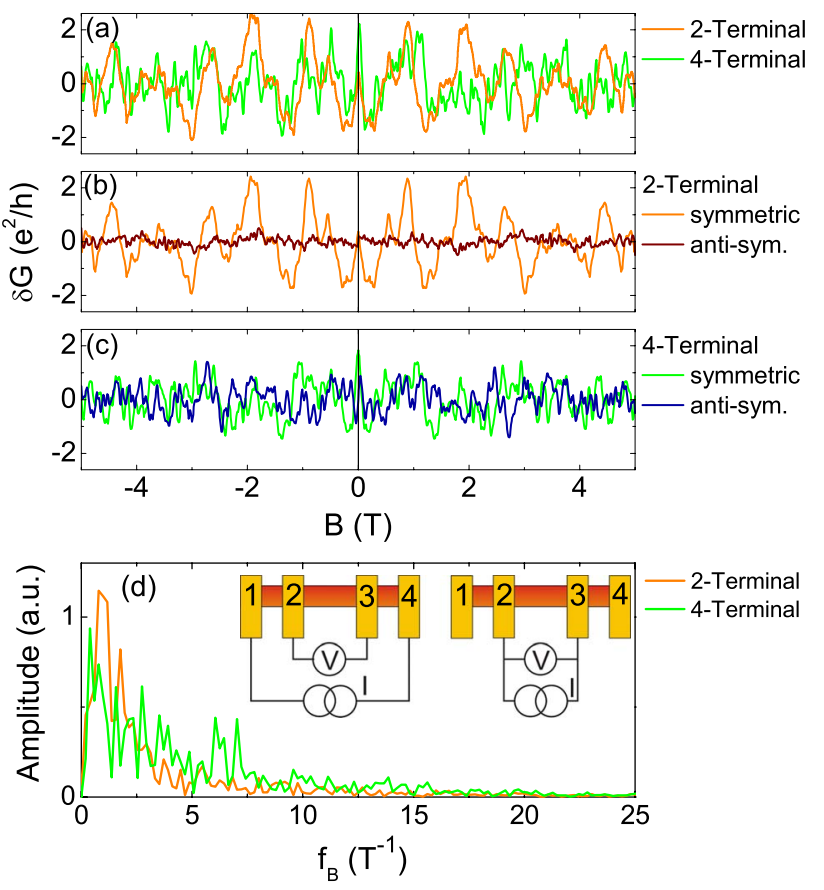

FIG. 3. (Color online) Conductance fluctuations $\delta G$ of sample A in units of $e^{2} / h$ as a function of magnetic field at $0.5 \mathrm{~K}$ : (a) comparison of the fourterminal and two-terminal magnetoconductances; (b) symmetric and antisymmetric contributions of the conductance fluctuations measured in a twoterminal configuration; (c) symmetric and antisymmetric contributions of the corresponding four-terminal configuration; and (d) fast Fourier transforms of the conductance fluctuations. The inset shows the schematics of the four-terminal (left) and two-terminal (right) measurement configurations.

ing of the energy window of the states contributing to the transport, which can be related to the thermal length $l_{T}$ $=\sqrt{\hbar \mathcal{D} / k_{B} T}$. Second, to the small energy transfer phasebreaking (Nyquist contribution) being the most relevant mechanism at low temperatures. Theoretically, for this process, the corresponding phase-coherence length $l_{\phi}$ is expected to show a temperature dependence proportional to $T^{-1 / 3}$. $^{14}$

Owing to the limited length of undoped MBE grown InN nanowires, usually the transport measurements are restricted to two-terminal configurations. ${ }^{6,15}$ In contrast, for doped InN wires, lengths exceeding $2 \mu \mathrm{m}$ are achieved which allow measurements in a four-terminal configuration. Thus the conductance fluctuations measured in both configurations can be compared directly. In order to achieve this for the twoterminal measurements [see inset of Fig. 3(d)], the contact resistances of contacts 2 and 3 were first subtracted from the total resistance before the two-terminal conductance of the wire segment was obtained. As can be seen in Fig. 2(a), the amplitudes and temperature dependence of $\operatorname{rms}(\delta G)$ are very similar, indicating that in the two-terminal configuration reliable information on the transport properties of the wire itself can be obtained.

The conclusions drawn from the temperature dependence of the mean fluctuation amplitude are supported by the measurements of the correlation field $B_{c}$ shown in Fig. 2(b). The latter is defined by $F\left(B_{c}\right)=\frac{1}{2} F(0)$ with $F(\Delta B)$ as the correlation function $F(\Delta B)=\langle\delta G(B+\Delta B) \delta G(B)\rangle{ }^{13}$ Here, $\langle\ldots\rangle$ represents the average over the magnetic field. For onedimensional conductors with $l_{\phi}>d, B_{c}$ is expected to be inversely proportional to the phase-coherence length: $B_{c}$ 
$=\gamma \Phi_{0} / l_{\phi} d$, with $\Phi_{0}=h / e$ as the magnetic flux quantum and $\gamma=0.95$ for $l_{\phi} \gg l_{T}{ }^{13,16}$ Indeed, as can be seen in the measurements shown in Fig. 2(b), above $1.5 \mathrm{~K} B_{c}$ increases proportional to $T^{0.19}$. Thus the corresponding decrease in $l_{\phi}$ with $T^{-0.19}$ [c.f. Fig. 2(c)] is close to the theoretically expected decrease in $l_{\phi} \sim T^{-1 / 3}$ for Nyquist dephasing. ${ }^{14}$ Interestingly, $l_{\phi}$ determined here is comparable to the length of the wire segment between contacts 2 and 3 for low $T$, supporting the statement given above that probably at temperatures below $1.2 \mathrm{~K}$, the transport within this segment is completely phase coherent. As can be seen in Fig. 2(c), for $T>1 \mathrm{~K}$, the thermal length $l_{T}$ determined from $\mathcal{D}$ is lower than $l_{\phi}$. For comparison the corresponding values of $B_{c}$ and $l_{\phi}$ of sample B are plotted in Figs. 2(b) and 2(c), showing a consistent behavior with the data of sample A.

The values of $l_{\phi}$ obtained from $B_{c}$ of sample A were used to calculate $\operatorname{rms}(\delta G)$ following the expression derived by Beenakker and van Houten: ${ }^{16}$

$$
\operatorname{rms}(\delta G)=\beta \frac{e^{2}}{h}\left(\frac{l_{\phi}}{L}\right)^{3 / 2}\left[1+\frac{9}{2 \pi}\left(\frac{l_{\phi}}{l_{T}}\right)^{2}\right]^{-1 / 2},
$$

with $\beta$ predicted to be $\sqrt{6}$. As can be seen in Fig. 2(a), a good agreement between the calculated and measured $\operatorname{rms}(\delta G)$ values is achieved, however with $\beta$ being smaller by a factor of about 4. A possible reason for the discrepancy regarding $\beta$ is the different geometrical situation, i.e., in the model a confined two-dimensional electron was considered. Generally, the experimentally obtained temperature dependence of $\operatorname{rms}(\delta G)$ of $T^{-0.84}$ shows a good agreement with the theoretical dependence proportional to $T^{-2 / 3}$ expected from Eq. (1) for the limit $l_{\phi} \gg l_{T}$.

According to the Onsager relations, the two-terminal magnetoconductance should be symmetric under magnetic field reversal. This is indeed the case as can be seen in Fig. 3(a). In Fig. 3(b) the magnetoconductance is decomposed in a symmetric $[\delta G(B)+\delta G(-B)] / 2$ and antisymmetric $[\delta G(B)+\delta G(-B)] / 2$ contribution. As a matter of fact, the antisymmetric contribution is close to 0 in the complete magnetic field range, while the symmetric contribution matches with the total magnetic conductance. More quantitatively, one finds a rms value of the conductance fluctuations normalized to $e^{2} / h$ of 0.96 for the symmetric part and a corresponding value of 0.14 for the antisymmetric contribution. For the four-terminal measurement configuration the situation is completely different, i.e., the symmetry under magnetic field reversal is lost [c.f. Fig. 3(a)]. As can be seen in Fig. 3(c), now the amplitudes of the symmetric and antisymmetric contributions of the magnetoconductance are comparable. This is also confirmed by the rms value of the normalized conductance, which are 0.65 and 0.47 for the symmetric and antisymmetric contributions, respectively. Apart from the different symmetry in the two measurement configurations, one can observe in Fig. 3(a) that in the four-terminal configuration the magnetoconductance fluctuations possess components of higher frequency compared to the two-terminal case. This is confirmed by the fast Fourier transformations shown in Fig. 3(d). The spectrum belonging to the fourterminal measurement contains components above $5 T^{-1}$, while for the two-terminal configuration only components below that frequency are found. The higher frequencies for the four-terminal configuration is probably due to the fact that here, an average voltage under the entire contacts is probed, while in the two-terminal configuration a smaller area restricted by the inner boundaries of the inner contacts is relevant. The fact that in a four-terminal configuration an antisymmetric contribution and higher frequency contributions are found is probably caused by the relatively weak coupling of the wire to the inner voltage probes.

In summary, phase-coherence in $n$-type doped $\mathrm{InN}$ nanowires was investigated in a four-terminal configuration. It is found that the decrease in the phase-coherence length with temperature can be attributed to small energy transfer processes. Owing to the four-terminal configuration, the symmetry of the conductance under field reversal is lost. Due to the large phase-coherence length found here, InN nanowires are interesting candidates for nanodevices based on electron interference.

${ }^{1}$ C. Thelander, P. Agarwal, S. Brongersma, J. Eymery, L. F. Feiner, A. Forchel, M. Scheffler, W. Riess, B. J. Ohlsson, U. Gösele, and L. Samuelson, Mater. Today 9, 28 (2006).

${ }^{2}$ W. Lu and C. M. Lieber, J. Phys. D 39, R387 (2006).

${ }^{3}$ S. De Franceschi, J. A. van Dam, E. P. A. M. Bakkers, L. Feiner, L. Gurevich, and L. P. Kouwenhoven, Appl. Phys. Lett. 83, 344 (2003).

${ }^{4}$ C. Fasth, A. Fuhrer, M. T. Bjork, and L. Samuelson, Nano Lett. 5, 1487 (2005).

${ }^{5}$ A. Pfund, I. Shorubalko, R. Leturcq, and K. Ensslin, Appl. Phys. Lett. 89, 252106 (2006).

${ }^{6}$ C. Blömers, Th. Schäpers, T. Richter, R. Calarco, H. Lüth, and M. Marso, Appl. Phys. Lett. 92, 132101 (2008).

${ }^{7}$ T. Richter, Ch. Blömers, H. Lüth, R. Calarco, M. Indlekofer, M. Marso, and Th. Schäpers, Nano Lett. 8, 2834 (2008).

${ }^{8}$ C. H. Liang, L. C. Chen, J. S. Hwang, K. H. Chen, Y. T. Hung, and Y. F. Chen, Appl. Phys. Lett. 81, 22 (2002).

${ }^{9}$ C.-Y. Chang, G.-C. Chi, W.-M. Wang, L.-C. Chen, K.-H. Chen, F. Ren, and S. J. Pearton, Appl. Phys. Lett. 87, 093112 (2005).

${ }^{10}$ T. Stoica, R. Meijers, R. Calarco, T. Richter, E. Sutter, and H. Lüth, Nano Lett. 6, 1541 (2006).

${ }^{11}$ F. Werner, F. Limbach, M. Carsten, C. Denker, J. Malindretos, and A. Rizzi, Nano Lett. 9, 1567 (2009).

${ }^{12}$ B. Al'tshuler, Pis'ma Zh. Eksp. Teor. Fiz. 41, 530 (1985); JETP Lett. 41, 648 (1985).

${ }^{13}$ P. A. Lee, A. D. Stone, and H. Fukuyama, Phys. Rev. B 35, 1039 (1987).

${ }^{14}$ B. L. Altshuler, A. G. Aronov, and D. E. Khmelnitsky, J. Phys. C 15 7367 (1982)

${ }^{15}$ C. Blömers, Th. Schäpers, T. Richter, R. Calarco, H. Lüth, and M. Marso, Phys. Rev. B 77, 201301 (2008).

${ }^{16}$ C. W. J. Beenakker and H. van Houten, Phys. Rev. B 37, 6544 (1988). 